07.2

\title{
Волновые эффекты в коаксиальном тракте при субнаносекундном переключении высоковольтного диода в режиме задержанного ударно-ионизационного пробоя
}

\author{
(С) М.С. Иванов, В.И. Брылевский, П.Б. Родин
}

Физико-технический институт им. А.Ф. Иоффее РАН, Санкт-Петербург, Россия

E-mail: m.ivanov@mail.ioffe.ru

Поступило в Редакцию 31 марта 2021 r.

В окончательной редакции 31 марта 2021 г.

Принято к публикации 2 апреля 2021 г.

Дан теоретический анализ волновых процессов в коаксиальном тракте при субнаносекундном переключении высоковольтного диодного обострителя импульсов. Установлены соотношения между амплитудами падающей, отраженной и прошедшей волн и измеряемыми в эксперименте напряжениями, а также между измеряемыми напряжениями и формой импульса генератора. Рассчитан эффект удвоения напряжения на диоде перед переключением. На основе полученных аналитических формул и экспериментальных данных определена временна́я зависимость коэффициента отражения обострителя и реконструированы волновые процессы в тракте при его переключении.

Ключевые слова: полупроводниковые приборы, ударная ионизация, субнаносекундные переключатели, коаксиальные тракты.

DOI: 10.21883/PJTF.2021.13.51119.18794

Явление задержанного ударно-ионизационного пробоя высоковольтных диодных $p^{+}-n-n^{+}$-структур состоит в субнаносекундном переключении диода из блокирующего в проводящее состояние $[1,2]$. Переключение происходит при быстром (>1kV/ns) увеличении обратного напряжения $V(t)$. В результате в последовательной нагрузке $R$ создается импульс тока с коротким (около $100 \mathrm{ps}$ ) передним фронтом. Явление лежит в основе работы диодного обострителя (ДО) импульсов уникального прибора мощной импульсной электроники субнаносекундного диапазона [2-5]. Продолжаются фундаментальные исследования и поиск путей улучшения параметров ДО [6-10].

Субнаносекундные времена переключения в сочетании с киловольтными напряжениями требуют применения волновых трактов [2,7], причем точные измерения могут быть осуществлены только в высококачественном коаксиальном тракте [7]. Во время переключения нестационарная проводимость ДО, представляющего собой сложную неоднородность коаксиального тракта, и амплитуда отраженной от этой неоднородности волны взаимно влияют друг на друга. Поэтому волновые свойства тракта оказывают определяющее влияние на процесс субнаносекундного переключения. Несмотря на очевидную важность проблемы, взаимодействие ДО с трактом до сих пор не было исследовано. Так, численное моделирование диодных обострителей (за исключением моделирования последовательного переключения приборов в сборке ДО [5]) проводилось для упрощенной цепи с сосредоточенными элементами. В настоящей работе рассмотрена простейшая модель, описывающая волно- вые процессы в коаксиальном тракте при субнаносекундном лавинном переключении диодного обострителя.

В эксперименте и практических приложениях $[2,5,7]$ ДО (или последовательная сборка из нескольких ДО) помещается в держатель, включенный в коаксиальный тракт. Такая схема эквивалентна подключению ДО в разрыв центральной жилы коаксиального тракта (рис. 1,a). Волновое сопротивление тракта равно сопротивлению нагрузки $R=50 \Omega$. На катод подается колоколообразный импульс обратного напряжения $V(t)$, амплитуда которого задается запускающим генератором. В эксперименте могут быть непосредственно измерены напряжение на нагрузке $U_{R}(t)$ и напряжение между катодом ДО и экраном кабеля $U_{C}(t)$, которое представляет собой суммарное напряжение на нагрузке и ДО [5,7]. Разность этих величин равна напряжению на ДО $U_{d}(t)$. Напряжение на катоде $U_{C}(t)$ измерялось в работах $[5,7]$. В работе [7] для этого применялся высококачественный резистивный ответвитель, который играл также роль держателя структуры (не показан на упрощенной схеме на рис. 1,a). Наличие дополнительной цепи для измерения напряжения на катоде $U_{C}(t)$ приводит к возмущению волновых процессов в содержащем ДО тракте, которое может быть минимизировано [7], но не исключено полностью. Далее мы пренебрегаем этим возмущением, когда используем данные эксперимента для иллюстрации полученных аналитических результатов. В то же время эти результаты без данной оговорки применимы к наиболее важной на практике схеме (рис. 1, $a$ ), в которой измерение $U_{C}(t)$ не производится. В дальнейшем анализе волновой тракт рассматривается как идеальная длинная линия. Учитывается только главная поперечная ТЕМ-мода. Ге- 

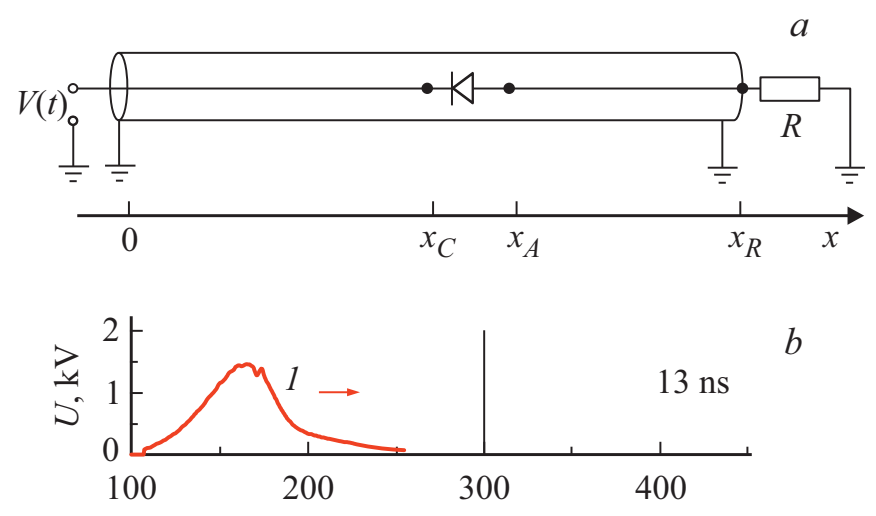

$b$
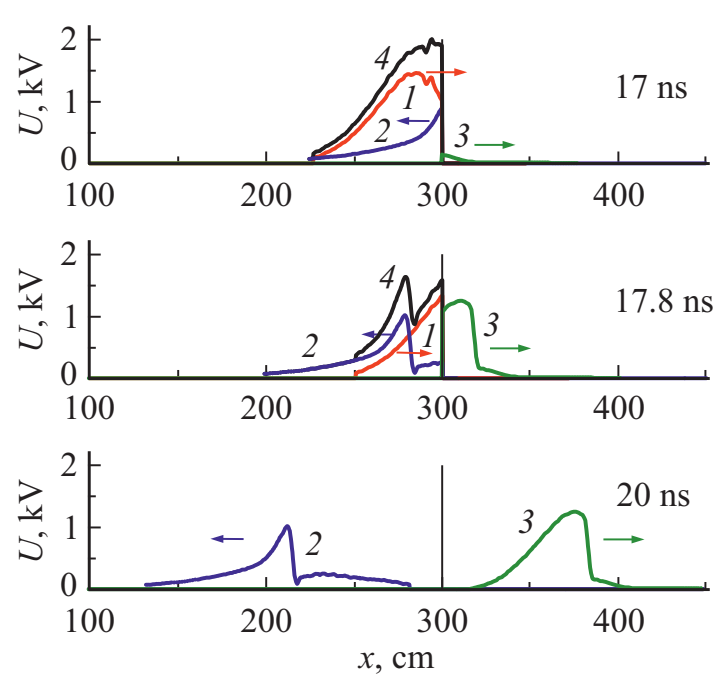

Рис. 1. Схема включения диодного обострителя в коаксиальный тракт (a) и распределения напряжения в тракте $(b)$. Диод помещен в разрыв центральной жилы нагруженного на согласованное сопротивление $50 \Omega$ кабеля между точками с координатами $x_{C}$ и $x_{A}$. Падающая $U_{i}(t, x)(1)$, отраженная $U_{r}(t, x)$ $(2)$ и прошедшая $U_{t}(t, x)(3)$ волны, а также мгновенная сумма $U(t, x)$ падающей и отраженной волн (4) реконструированы по данным эксперимента [7] в последовательные моменты времени $t=13.0,17.0,17.8,20.0 \mathrm{~ns}$. Стрелки указывают направление движения волны.

нерацией других пространственно-временны́х мод при отражении от диода пренебрегаем.

Рассмотрим связь между амплитудой электромагнитных импульсов в коаксиальном тракте и измеряемыми в эксперименте напряжениями $U_{C}(t)$ и $U_{R}(t)$. Распределение напряжения вдоль коаксиального тракта обозначим как $U(t, x)$, амплитуды падающей, отраженной и прошедшей волн - как $U_{i}(t, x), U_{r}(t, x), U_{t}(t, x)$ соответственно. Таким образом, $U(t, x)=U_{i}(t, x)+U_{r}(t, x)$ при $0<x<x_{C}, \quad U(t, x)=U_{t}(t, x)$ при $x_{C}<x<x_{R}$, $U_{C}(t)=U\left(t, x_{C}\right), \quad U_{R}(t)=U\left(t, x_{R}\right) \quad$ (рис. $\left.1, b\right)$. Падающая волна полностью задана импульсом генератора: $U_{i}(t, x)=V(t-x / c)$, где $c-$ скорость волны в тракте. Включенный в разрыв центральной жилы ДО представляет собой сложную неоднородность коаксиального тракта, в результате взаимодействия с которой па- дающая волна разделяется на отраженную и прошедшую [11]. Амплитуды этих волн слева и справа от неоднородности, т. е. в точках $x=x_{C}$ и $x=x_{A}$, связаны соотношением

$$
U_{i}\left(t, x_{C}\right)=U_{t}\left(t, x_{A}\right)+U_{r}\left(t, x_{C}\right)
$$

которое отвечает условию равенства единице суммы коэффициентов прохождения $T$ и отражения $R$. Уравнение (1) следует из условия сохранения электрического заряда на центральном электроде и выполняется в меру точности линейного соотношения между плотностью заряда и амплитудой волны. Необходимо также выполнение соотношения $W=x_{A}-x_{C} \ll c \delta$ (где $\delta-$ полуширина импульса [11]), которое позволяет отождествить точки $x_{A}$ и $x_{C}$ в правой части уравнения (1). При типичных значениях $\delta \sim 1 \mathrm{~ns}, W \sim 100 \mu \mathrm{m}$ это условие заведомо выполнено. Заметим, что следствием (1) является сохранение потока энергии с учетом мощности, рассеиваемой на диоде, но без учета потерь на высокочастотное излучение, связанное с трансформацией мод при отражении от диода.

Измеряемое в эксперименте напряжение между катодом ДО и экраном кабеля $U_{C}(t)$ представляет собой сумму напряжений на ДО и нагрузке и равно сумме амплитуд падающей и отраженной волн на катоде в точке $x=x_{C}$ :

$$
U_{C}(t) \equiv U\left(t, x_{C}\right)=U_{i}\left(t, x_{C}\right)+U_{r}\left(t, x_{C}\right)
$$

Из уравнений (1) и (2) следует соотношение

$$
U\left(t, x_{C}\right)+U_{t}\left(t, x_{A}\right)=2 U_{i}\left(t, x_{C}\right) .
$$

С помощью уравнений (1) и (3) находим напряжение на ДО $U_{d}(t) \equiv U_{C}(t)-U_{A}(t)$ :

$$
U_{d}(t)=2 U_{i}\left(t, x_{C}\right)-2 U_{t}\left(t, x_{A}\right)=2 U\left(t, x_{C}\right)-2 U_{i}\left(t, x_{C}\right) .
$$

Из уравнений (2) и (4) следует, что амплитуда отраженной от ДО волны в точке $x=x_{C}$ составляет половину напряжения на ДО

$$
U_{d}(t)=2 U_{r}\left(t, x_{C}\right)
$$

Амплитуды падающей $U_{i}\left(t, x_{C}\right)$ и прошедшей $U_{t}\left(t, x_{A}\right)$ волн на электродах ДО с учетом временно́й задержки могут быть представлены как $U_{i}\left(t, x_{C}\right)=V\left(t-x_{C} / c\right)$ и $U_{A}(t) \equiv U_{t}\left(t, x_{A}\right)=U_{R}\left[t+\left(x_{R}-x_{A}\right) / c\right]$. С учетом этих соотношений из уравнения (3) получаем

$$
U_{C}(t)+U_{R}\left[t+\left(x_{R}-x_{A}\right) / c\right]=2 V\left[t-x_{C} / c\right] .
$$

Таким образом, сумма напряжения на катоде ДО и напряжения на нагрузке оказывается равной удвоенной амплитуде импульса генератора $V(t)$, измеренного в режиме работы на согласованную нагрузку. На рис. 2 показаны экспериментальные зависимости $U_{C}(t)$ и $U_{R}(t)$ по данным работы [7] и их сумма. Зависимости (см. рис. 2 


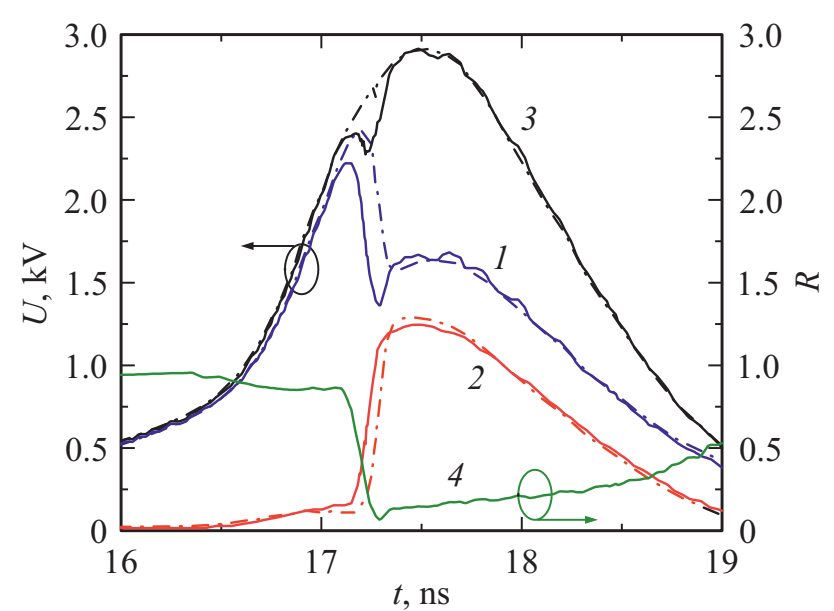

Рис. 2. Зависимости от времени напряжений на диоде и нагрузке $U_{C}(t)$ (кривая 1 ) и на нагрузке $U_{R}(t)$ (кривая 2) по данным эксперимента [7] (сплошные линии) и результатам численного моделирования (штрихпунктирные линии). Сумма $U_{C}(t)$ и $U_{R}(t)$ (кривая 3), согласно уравнению (6), соответствует удвоенному импульсу запускающего генератора $V(t)$, имеющему колоколообразную форму. 4 - зависимость от времени коэффициента отражения ДО $R(t)$.

в [7]) соответствуют диодной $p^{+}-n-n^{+}$-структуре с напряжением стационарного пробоя $U_{b}=1.1 \mathrm{kV}$ и площадью $1 \mathrm{~mm}^{2}$, изготовленной по диффузионной технологии из нейтронно-легированного кремния. Колоколообразный импульс генератора имел полуширину $1.5 \mathrm{~ns}$ [7]. Штрихпунктирными линиями на рис. 2 показаны зависимости $U_{C}(t)$ и $U_{R}(t)$ и их сумма, полученные путем численного моделирования переключения ДО в коаксиальном тракте с помощью TCAD Silvaco (результаты моделирования будут представлены отдельно). Как для экспериментальных, так и для расчетных кривых сумма с разумной точностью представляет собой колоколообразный импульс полушириной $1.5 \mathrm{~ns}$ и амплитудой около $3 \mathrm{kV}$. Значительное отклонение от колоколообразной формы для экспериментальных зависимостей отражает неидеальность тракта в эксперименте, в том числе обусловленную наличием резистивного ответвителя [7], необходимого для измерения напряжения в точке $x=x_{C}$. Таким образом, соотношение (3) позволяет восстановить форму импульса генератора без непосредственного измерения на согласованной нагрузке.

Из уравнения (6) следует, что до момента начала переключения напряжение на катоде $U_{C}$ почти в 2 раза превышает амплитуду импульса генератора. Этот эффект связан со сложением падающей и отраженной волн. При возрастании тока в нагрузке напряжение на катоде падает, поскольку амплитуда импульса генератора задана. Известно, что переключение ДО в режиме задержанного лавинного пробоя происходит, когда напряжение на приборе примерно вдвое превосходит напряжение стационарного лавинного пробоя $U_{b}[1,2,7]$. Описанный выше эффект удвоения напряжения означает, что переключение диода в волновом тракте может быть достигнуто для запускающего импульса $V(t)$, амплитуда которого лишь незначительно превосходит $U_{b}$.

Установим связь между экспериментально измеряемыми величинами $U_{C}(t)$ и $U_{R}(t)$ и пространственновременны́ми амплитудами импульсов в тракте. Для амплитуд волн в идеальной линии выполняются соотношения

$$
\begin{gathered}
U_{i}(t, x)=U_{i}\left[t+\left(x_{C}-x\right) / c, x_{C}\right], \\
U_{r}(t, x)=U_{r}\left[t-\left(x_{C}-x\right) / c, x_{C}\right], \\
U_{t}(t, x)=U_{R}\left[t+\left(x_{R}-x\right) / c\right] .
\end{gathered}
$$

Учитывая уравнения (1), (2) и полагая $x_{A}=x_{C}$ (в силу $\left.W=x_{A}-x_{C} \ll c \delta\right)$, получаем

$$
\begin{gathered}
2 U_{i}(t, x)=U_{C}\left[t+\left(x_{C}-x\right) / c\right]+U_{R}\left[t+\left(x_{R}-x\right) / c\right], \\
2 U_{r}(t, x)=U_{C}\left[t-\left(x_{C}-x\right) / c\right] \\
-U_{R}\left[t+\left(x_{R}+x-2 x_{C}\right) / c\right] .
\end{gathered}
$$

Полное напряжение $U(t, x)=U_{i}(t, x)+U_{r}(t, x)$ в тракте между генератором и ДО и временны́е зависимости для коэффициентов отражения и прохождения

$$
\begin{aligned}
& R(t)=U_{r}\left(t, x_{c}\right) / U_{i}\left(t, x_{c}\right), \\
& T(t)=U_{t}\left(t, x_{A}\right) /\left(U_{i}\left(t, x_{c}\right)\right.
\end{aligned}
$$

могут быть явно выражены через $U_{C}(t)$ и $U_{R}(t)$ с помощью уравнений (7)-(10).

Коэффициент отражения $R(t)$, построенный с помощью уравнений (7)-(10) по данным эксперимента [7], показан на рис. 2 (кривая 4). Резкое уменьшение коэффициента отражения при 100-пикосекундном переключении обострителя оказывает решающее влияние на динамику электромагнитных импульсов в тракте.

Пространственно-временны́е зависимости амплитуд волн в тракте, вычисленные с помощью полученных выше соотношений (7)-(9), и измеренных в экспериментах [7] напряжений $U_{C}(t)$ и $U_{R}(t)$ (рис. 2) представлены на рис. $1, b$. ДО находится в точке $x_{C}=300 \mathrm{~cm}$; $x_{R}=600 \mathrm{~cm},\left(x_{A}-x_{C}\right) \ll x_{C}, x_{A}, x_{R}$. До переключения падающая волна почти полностью отражается, так как коэффициент отражения $R$ близок к единице (рис. 2). Ток в нагрузке незначителен. На этой фазе процесса сложение падающей и отраженной волн почти удваивает напряжение на ДО по сравнению с амплитудой импульса генератора $V(t)$. В процессе переключения ДО коэффициент отражения быстро уменьшается вследствие увеличения проводимости прибора. Это приводит к резкому уменьшению амплитуды отраженной волны. Как следствие, напряжение на катоде $U_{C}$ значительно и резко уменьшается. Одновременно нарастает амплитуда прошедшей волны, т.е. ток в нагрузке. Заметим, что значительная величина $x_{R}$ выбрана для наглядности представления результата. При длине тракта около $100 \mathrm{~cm}$ принципиально возможно отражение волны $U_{r}$ от генератора. Однако в эксперименте [7] конструкция 
выходного каскада генератора обеспечивает поглощение этой волны.

Таким образом, в работе установлена связь между измеряемыми в эксперименте напряжениями и запускающим импульсом генератора, определена временна́я зависимость коэффициента отражения ДО при субнаносекундном ударно-ионизационном переключении, а также реконструированы профили падающей, отраженной и прошедшей волн в коаксиальном тракте.

\section{Благодарности}

Авторы признательны А.Ф. Кардо-Сысоеву за полезные обсуждения.

\section{Конфликт интересов}

Авторы заявляют, что у них нет конфликта интересов.

\section{Список литературы}

[1] И.В. Грехов, А.Ф. Кардо-Сысоев, Письма в ЖТФ, 5 (15), 950 (1979).

[2] A.F. Kardo-Sysoev, in Ultra-wideband radar technology, ed. by J.D. Taylor (CRS Press, Boca Raton-London-N.Y.Washington, 2001), p. 205.

[3] R.J. Focia, E. Schamiloglu, C.B. Fledermann, F.J. Agee, J. Gaudet, IEEE Trans. Plasma Sci., 25 (2), 138 (1997). DOI: $10.1109 / 27.602484$

[4] I.V. Grekhov, IEEE Trans. Plasma Sci., 38 (5), 1118 (2010). DOI: $10.1109 /$ TPS.2010.2043857

[5] С.К. Любутин, С.Н. Рукин, Б.Г. Словиковский, С.Н. Цыранов, Письма в ЖТФ, 31 (5), 36 (2005). [Пер. версия: 10.1134/1.1894430]; ФТП, 44 (7), 962 (2010). [Пер. версия: 10.1134/S1063782610070171].

[6] L.M. Merensky, A.F. Kardo-Sysoev, D. Shmilovitz, A.M. Kesar, IEEE Trans. Plasma Sci., 42 (12), 4015 (2014). DOI: 10.1109/TPS.2014.2366551

[7] V. Brylevskiy, I. Smirnova, A. Gutkin, P. Brunkov, P. Rodin, I. Grekhov, J. Appl. Phys., 122 (18), 185701 (2017). DOI: $10.1063 / 1.5004524$

[8] P. Rodin, M. Ivanov, J. Appl. Phys., 127 (4), 044504 (2020). DOI: $10.1063 / 1.5097831$

[9] A.S. Kesar, A. Raizman, G. Atar, S. Zoran, S. Gleizer, Y. Krasik, D. Cohen-Elias, Appl. Phys. Lett., 117 (1), 013501 (2020). DOI: $10.1063 / 5.0016228$

[10] C.C. Herrmann, J. Croman, S.V. Baryshev, arXiv: 2011.13081 (2020).

[11] P.W. Smith, Transient electronics: pulsed circuit technology (John Wiley \& Sons, Chichester, 2002). 